\title{
Age and sex differences in children's spatial search strategies
}

\author{
MARCIA L. SPETCH \\ University of Alberta, Edmonton, Alberta, Canada \\ and \\ MARISE B. PARENT \\ Georgia State University, Atlanta, Georgia
}

\begin{abstract}
Male and female children, 3, 4, and 5 years old, searched for a sticker that was hidden in 1 of 15 linearly aligned boxes. Two identical bear-shaped landmarks cued the sticker location, which was always in the middle of 3 boxes that separated the two landmarks. The absolute locations of the landmarks and sticker varied across training trials, but the distance in relation to each other remained constant. Training continued until the child chose the correct box first for 3 consecutive trials or for a maximum of 20 trials. Striking age and sex differences emerged in acquisition: The percentage of children who reached criterion increased over age groups to $100 \%$ for the boys but stayed at approximately $20 \%$ for the girls. A landmark expansion test (with the landmarks moved farther apart) given to children who met criterion revealed that most of these children chose the middle location.
\end{abstract}

Using visual landmarks to find a hidden goal is common in both human and nonhuman animals, but the strategies used to derive spatial information from landmarks can vary across species (see Spetch \& Kelly, 2006). One situation in which differences between species have been found is when a goal is hidden in the middle of an array of identical landmarks that maintain a constant distance from each other but are moved within the search space across training trials. Spetch, Cheng, and MacDonald (1996) and Spetch et al. (1997) found that adult humans responded differently than pigeons on "expansion" tests in which the landmarks were spread farther apart: In both computer touch-screen tasks and open-field tasks, adult humans consistently responded to the middle of the expanded array, suggesting a relational use of the landmarks, whereas pigeons searched at the trained distance from individual landmarks, suggesting an absolute strategy. Gerbils (Collett, Cartwright, \& Smith, 1986) and nonhuman primates (MacDonald, Spetch, Kelly, \& Cheng, 2004; Poti, Bartolommei, \& Saporiti, 2005; Sutton, Olthof, \& Roberts, 2000) also have not responded to the middle of the array on expansion tests. The difference between adult humans and these nonhuman species appears to reflect

This research was supported by a Natural Sciences and Engineering Research Council of Canada discovery grant to M.L.S. We thank Danny Darby, Curriculum Director of the Capitol Hill Child Enrichment Center and the Lanette Suttles Child Development Center at Georgia State University, for facilitating this research, and we also thank the parents and children of the centers for their participation. We thank Isaac Lank for his assistance with the design and construction of our apparatus, Ryan Earley for statistical advice, and Amy Ross and Alexandra Twyman for comments on the manuscript. Correspondence concerning this article should be sent to M. L. Spetch, Department of Psychology, BSP 217, University of Alberta, Edmonton, AB, T6G 2E9 Canada (e-mail: mspetch@ualberta.ca). "preferred" strategy rather than "ability," because both pigeons and Clark's nutcrackers can learn to respond in the middle of a landmark array if the training task cannot be solved on the basis of absolute distances (J. E. Jones, Antoniadis, Shettleworth, \& Kamil, 2002; Kamil \& J. E. Jones, 1997).

MacDonald et al. (2004) reported that human children also responded differently than human adults to landmark expansion tests. In one task, the search space consisted of an array of discrete hiding places, and the goal was in the middle of, and directly adjacent to, the four identical landmarks in training. Children (ages 3-7) and adults rapidly learned to choose the hiding place in the middle of the landmarks on training trials, but they responded very differently to expansion tests. Whereas all adults continued to choose the middle, only 1 out of 13 children chose the middle. The remaining children searched almost exclusively in hiding places adjacent to the landmarks, suggesting that they had used the landmarks as beacons. To determine how children would respond in a task that could not be solved with a beaconing strategy, MacDonald et al. tested children (ages 3-5) on another task in which the landmarks were farther from the goal. This search space was a tray filled with confetti, and the goal was a sticker hidden in the middle of, but a fixed distance away from, four identical landmarks. This task proved difficult for children to learn, and only 10 out of 19 children reached criterion within 20 trials. Of the 10 children who learned, 3 searched closest to the middle on the expansion test, suggesting a relational strategy. Most of the remaining children searched closer to the learned distance and direction from individual landmarks, suggesting an absolute strategy. Together, these results by MacDonald et al. indicate that the ease with which children learn, and the 
strategy they display on expansion tests, depends on the task. However, in neither task did the children show a consistent tendency to search in the middle on expansion tests, indicating that their preferred strategies differ from those of adults.

The contrast between how children in MacDonald et al. (2004) responded to expansion tests and how adults responded to these tests (MacDonald et al., 2004; Spetch et al., 1996, 1997) is interesting and warrants further experimental attention. Our study extends this research in several ways. First, our study provides another assessment of strategies used by children in a task that cannot be learned using a beaconing strategy. Because children in Experiment 2 of MacDonald et al. had difficulty learning the task, we attempted to develop a task that would be easier for children to learn. Like the first task used by MacDonald et al. (which children learned rapidly), we used discrete hiding places, but unlike in their first task, our landmarks were not directly adjacent to the goal. In addition, we reduced the search space to a single linear array of hiding places, which we thought might also make the task easier. We sought to determine whether young children would readily find a goal that is in the middle of, but not directly adjacent to, identical landmarks, and if so, whether they would consistently choose the middle location on expansion tests.

Second, our study was designed to compare children at each of three age groups $(3,4$, and 5 years) to examine developmental trends in ease of learning the task or strategy used. No clear developmental trends were apparent in Experiment 2 of MacDonald et al. (2004), but their numbers were too small to be conclusive. We expected that older children would learn the task more readily than younger children. However, it was not clear whether to expect age differences in the strategy used on expansion tests, because previous literature leads to conflicting predictions. Specifically, the finding that adult humans show a strong preference for a relational strategy, whereas nonhumans prefer an absolute strategy, suggests that older children might be more likely than younger children to select the middle location on the test. However, an opposite prediction can be derived from studies of infants' use of spatial cues to determine object size (see, e.g., Duffy, Huttenlocher, \& Levine, 2005), which have found that attention to relative spatial cues developmentally precedes attention to absolute cues.

Finally, our study compared boys and girls to determine whether there were any sex differences in performance on our task. Experiment 2 of MacDonald et al. (2004) did not allow a determination of sex differences because there were only 4 girls in their study, and only 1 of those girls learned the task. Sex differences in spatial ability are often seen in both humans and nonhumans, but these differences tend to emerge after puberty, except in mental rotation tasks (see reviews by C. M. Jones, Braithwaite, \& Healy, 2003; Voyer, Voyer, \& Bryden, 1995). Thus, on the basis of prior literature, we were not expecting to see sex differences in the performance of preschool children on a seemingly simple spatial task.

\section{METHOD}

\section{Participants}

Twelve 3-year-olds (mean age $=43$ months, range $=36-46$ months), fifteen 4 -year-olds (mean age $=54$ months, range $=48-59$ months), and eleven 5-year-olds (mean age $=61$ months, range $=$ 60-65 months) completed the study. For the three age groups, there were 6,9 , and 5 girls and 6,6 , and 6 boys, respectively. The children were recruited from two daycare centers in Atlanta, through letters sent to parents. The Georgia State University Institutional Review Board and the University of Alberta Human Ethics Committee approved the recruitment and experimental procedures. All of the parents signed a consent form on behalf of their children. The children were asked if they wanted to "play the game" after it was explained to them, and they were told that they could stop playing any time they wanted to. Stickers were provided to the daycare to be distributed among the children who did not participate.

\section{Materials}

The search space was a linear array of 15 identical white Plexiglas boxes spaced $0.5 \mathrm{~cm}$ apart and attached to a Plexiglas base (see Figure 1). Each box was $2.5 \mathrm{~cm} \times 5 \mathrm{~cm} \times 3.5 \mathrm{~cm}$ and had a flat lid that was $5.3 \mathrm{~cm} \times 6.5 \mathrm{~cm}$. The lip of the lid protruded beyond the box so that it could be lifted easily. The landmarks were two red plastic teddy bears, $3.8 \mathrm{~cm}$ high; the goal was a sticker.

\section{Procedure}

The children were tested individually in a private room in the daycare centers. Each child was given a total of 22 stickers, whether or not they found them. At the beginning of the experiment, each child was brought into the testing room by Experimenter 1 and was introduced to Experimenter 2. The child sat at a small table facing the array of boxes. Experimenter 1 sat beside the child and Experimenter 2 sat across from the child. The teddy bear landmarks were placed facing one another on boxes that were spaced with three boxes in between them. The children were asked if they wanted to play a game of hide and seek for stickers, and all of the children agreed. They were then asked to choose one of three sets of stickers to use in the game. Then, while the children watched, Experimenter 2 placed a sticker in the center box between the bears and closed the lid. Experimenter 1 told the children to find the sticker and informed them

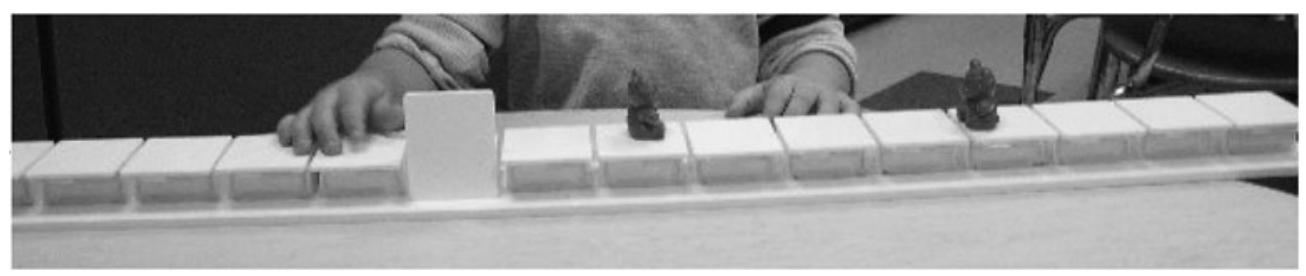

Figure 1. Photograph of the linear search array. The toy teddy bears served as the landmarks. 
that the sticker would never be in the boxes under the bears, but that the bears could help them find the sticker.

Training. For all of the remaining trials, Experimenter 1 told the children to turn around and close their eyes while Experimenter 2 hid the sticker. The bears were moved so that the hiding location was always in the center of the three boxes between the bears. The lids on several boxes were lifted and closed so that auditory cues could not signal the goal box. Experimenter 1 then told the children to turn around and look for the sticker, and told them, "Remember, the bears can help you find the sticker." The children were told to open only one box at a time and to close the lid before looking in another box, and were reminded as needed. Experimenter 2 recorded the order of each choice and any task-related spontaneous comments made by the children. The children were given as many choices as required to find the sticker, and when they opened the correct box, Experimenter 1 said, "Ta da, you found it." The children removed and kept each sticker found. Training continued for a maximum of 20 trials or until the children found the sticker on their first choice on 3 consecutive trials. After the 20 trials or after the acquisition criterion was reached, Experimenter 1 asked the children, "How do the bears help you find the sticker? What are the bears doing?" and Experimenter 2 recorded the responses. Children who did not meet criterion were thanked for playing and were given any remaining stickers.

Testing. A single test trial was given to children who met the training criterion. The bears were placed five boxes apart, and no sticker was placed in any of the boxes. The children were allowed to make five choices, and then Experimenter 2 gave them the sticker and said that she had forgotten to hide it. The children were thanked for playing and were given any remaining stickers.

\section{RESULTS}

\section{Acquisition}

The task was surprisingly difficult for the children. Of the 38 children, only 14 met criterion within the 20 training trials. Figure 2 shows mean trials to criterion for boys and girls in each of the three age groups. Children who did not reach criterion were given a score of 20 . These data suggest an interaction between age and sex, with a clear sex dif-

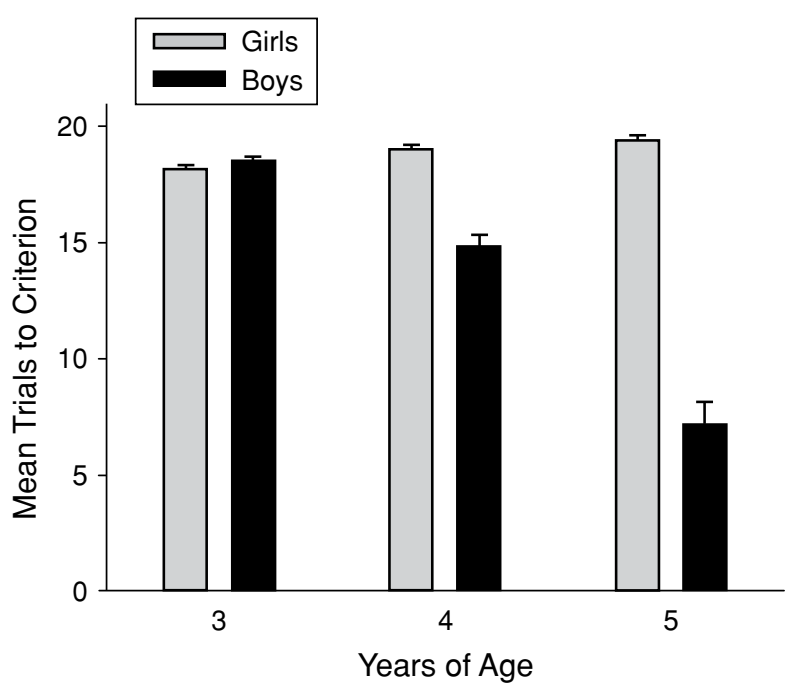

Figure 2. Mean trials to an accuracy criterion for boys and girls at each of the three age groups. Error bars indicate standard error of the mean. ference emerging in the older children. Because variance differed substantially across groups, we analyzed the data using separate nonparametric Kruskal-Wallis tests to examine age and gender effects. These tests revealed significant effects of both age $\left(\chi_{2}^{2}=6.26, p=.044\right)$, and gender $\left(\chi_{1}^{2}=6.95, p=.008\right)$. The emergence of a sex difference in the older children was also reflected in the percentage of children who reached criterion. The percentage of boys who reached criterion increased substantially over the three age groups $(17 \%, 50 \%$, and $100 \%$ for the $3-$, $4-$, and 5 -year-olds, respectively), but the percentage of girls who reached criterion was similar across age groups $(17 \%$, $22 \%$, and $20 \%$ ). Fisher's exact test $(p<.05)$ revealed no significant sex difference in the 3- and 4-year-old groups, but the percentage of 5-year-old boys who reached criterion was significantly greater than the percentage of 5-year-old girls who reached criterion.

Children who did not reach criterion showed some interesting tendencies in their choice behavior. One tendency was to start at one end and search through the boxes in order. To quantify this "serial search" tendency, we determined the proportion of trials (out of 20) on which the child started at either end and chose adjacent boxes in one direction without skipping any except the landmark box until finding the sticker. Another tendency was to search in boxes adjacent to the landmarks, either on the correct side or on either side of the landmarks. To quantify these patterns, we determined the proportion of trials on which the first choice was to a box adjacent to a landmark and between the landmarks or adjacent to a landmark but outside of the landmark array. Finally, we scored the proportion of trials on which the first choice was to the correct middle location. These four measures are shown in Figure 3 for the boys and girls in each age group who did not meet criterion (all of the 5-year-old boys met criterion). Several interesting trends were apparent. First, none of the children showed a high proportion of middle choices. Second, 3-year-old boys and 4-year-old girls showed a strong serial search tendency: This pattern occurred on $47 \%$ and $52 \%$ of the trials, respectively. Third, only the 5-year-old girls showed a strong tendency to search in adjacent boxes on the correct side of the landmarks: $88 \%$ of their adjacent box choices were on the correct side, which was significantly above chance $[50 \%, t(3)=6.66, p<.001]$. An ANOVA showed a significant effect of age on "adjacent between" choices for girls $[F(2,13)=37.0, p<.001]$. There were no significant effects of age on the other measures for girls or on any measures for boys (all $p s>.1$ ).

\section{Expansion Test}

Fourteen children ( 4 girls and 10 boys) reached criterion and were given the expansion test. Nine children ( 7 boys and 2 girls) chose the center location first, and the remaining children ( 3 boys and 2 girls) chose the location that maintained a correct absolute distance from one of the landmarks (see Table 1, top row). A chi-square test comparing the observed choices of the center and absolute locations ( 9 and 5) to the expected frequencies based on random choice (4.7 and 9.3) revealed a significant pref- 

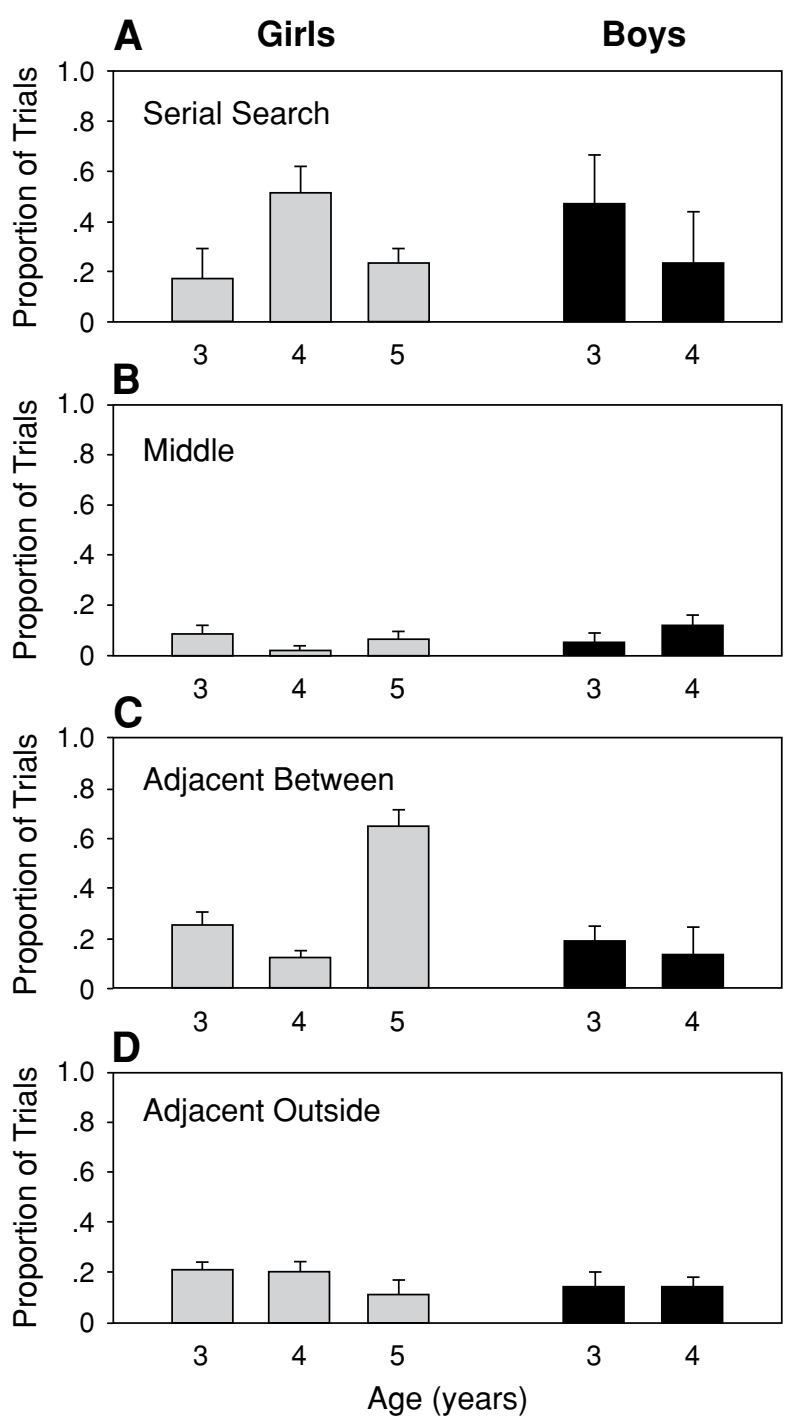

Figure 3. Mean proportion of trials on which boys and girls in each age group searched with a serial pattern (A), or in which the first choice was to the box in the middle of the landmarks (B), to a box between the landmarks but adjacent to one of them (C), or adjacent to a landmark but outside of the array (D). Only the children who did not meet the accuracy criterion are included (there were no 5-year-old boys who failed to reach criterion). Error bars indicate standard error of the mean.

erence for the middle location $\left[\chi^{2}(1)=6.02, p<.02\right]$. The bottom row of Table 1 shows the frequency of total choices (five per child) in each location. All of the children except 2 made all of their choices to boxes between the landmarks. The observed frequency of choosing boxes between the landmarks and outside of the landmarks (64 and 6 , respectively) differed significantly from expected frequencies of 26.9 and $43.1\left[\chi^{2}(1)=83.1, p<.001\right]$. Thus, the children who reached criterion clearly learned to use the landmarks, and most chose the middle on the expansion test.

\section{Responses to Landmark Questions}

Only two children (both 5-year-old boys) responded with the word "middle," and one 5-year-old boy said "between." Responses of all of the remaining children who chose the middle were nonspecific (e.g., "I found it"; "Looking in the boxes"; "They're there"; "Thinking, finding them").

\section{DISCUSSION}

There are several notable results in this study. First, this task involving discrete hiding places was not easier for children to learn than the continuous space task used by MacDonald et al. (2004). Only 37\% of the children reached our accuracy criterion within 20 training trials. Second, of the children who met our accuracy criterion, most (64\%) chose the middle location first on expansion tests. The third, and perhaps most striking result, was a sizable difference in task acquisition across age groups and sex. Most of the 3-year-old boys and most of the girls in all three age groups failed to reach criterion. By 5 years of age, all of the boys learned the task readily.

Children in our task showed a lower success rate in acquisition but a higher tendency to choose the middle location on the expansion test in comparison with children in Experiment 1 of MacDonald et al. (2004). We suspect that the critical difference was in whether a beaconing strategy would allow children to reach criterion. In MacDonald et al.'s study, a tendency to search near the landmarks could produce accurate responding during training because the goal was directly adjacent to all four landmarks and therefore might be the most attractive location to search. This beaconing would produce a tendency to search near the landmarks on the test. In our study, beaconing would prevent children from reaching criterion, and hence from proceeding to the test. Interestingly, the search patterns of 5-year-old girls who did not reach criterion suggested that they had learned that the sticker was between the landmarks, but they nevertheless showed a strong tendency to search next to a landmark.

Our finding that most of the children who acquired the task responded to the middle location on the expansion test contrasts with the results obtained in Experiment 2 of MacDonald et al. (2004), in which children were more likely to use an absolute strategy. We do not know why this is the case. Two other recent studies using different kinds of spa-

Table 1

First Choice and Total Choices (Five per Child) on the Expansion Test for the 14 Children who Met Criterion

First Choice

\begin{tabular}{|l|l|l|l|l|l|l|l|l|l|l|l|l|l|l|}
\hline & & & & L & & 1 & 9 & 4 & & L & & & & \\
\hline
\end{tabular}

Total Choices

Note $-\mathbf{L}$ indicates the landmark locations. Choices are shown relative to the landmark locations, which were separated by 5 boxes during the test. The actual location of the landmarks within the array of 15 boxes varied across children. Some children chose a location more than once. 
tial tasks (Solomon \& Levine, 2005; Uttal \& Newcombe, 2005) also found that most 4-year-old children searched in the middle on expansion tests. Together, these results clearly indicate that preschool children sometimes search in the middle on expansion tests. However, this tendency is much less consistent in children than it is in adults.

In adult humans, searching in the middle on expansion tests has been assumed to reflect the use of an abstract relational rule in which the goal is learned in relation to the configuration of landmarks (see Cheng \& Spetch, 1998). Spontaneous comments by adult humans such as "it's in the middle," and transfer of learning to the same configuration composed of entirely new landmarks, support this interpretation (Spetch et al., 1996). However, responding "in the middle" on an expansion test can also result from less abstract processes. For example, honeybees sometimes show a relational use of landmarks that arises through an image-matching process (Cartwright \& Collett, 1983). In our study, choice of the middle could arise from averaging vectors from the two landmarks, provided that both landmarks were weighted equally (see Cheng \& Spetch, 1998, for a discussion of averaging). Interestingly, of the 9 children who chose the middle location on the test, only 3 (all 5 -year-old boys) used the words "middle" or "between." This raises the possibility that the remaining children did not use an abstract middle rule, or that they did, but could not verbalize it. Indeed, there is evidence that young children can sometimes know more than they can say, as evidenced by a mismatch between gestures and speech (see, e.g., Goldin-Meadow, 1997).

The striking sex differences in performance that we observed are interesting and surprising. A male advantage in spatial tasks has been found in many studies with both humans and nonhumans, but most often this advantage has been seen postpuberty (see C. M. Jones et al., 2003, for a review). Although a few studies have reported a male advantage in spatial ability in young children, the age at which this sex difference emerges appears to depend on the nature of the spatial ability tested (see Voyer et al., 1995). Specifically, sex differences have been found in preschool children, but these have primarily occurred in tasks that required mental transformations of objects, such as mental rotation (Rosser, Ensing, Gilder, \& Lane, 1984), 3-D puzzle tasks (McGuinness \& Morley, 1991), and spatial transformation tasks (Levine, Huttenlocher, Taylor, \& Langrock, 1999). In other kinds of spatial tasks, sex differences have typically not been seen in preschool children (see, e.g., Uttal, Gregg, Tan, Chamberlin, \& Sines, 2001; Vasilyeva \& Huttenlocher, 2004). For example, Choi and Silverman (2003) found that sex differences in route learning did not appear until 12 years of age.

The sex differences we observed could result from biological or sociocultural influences, or some combination of both (see Geary, 1996). Moreover, they could reflect differences in either spatial "ability" or "preferred" search strategy. We speculate that our task might have been particularly sensitive to early sex differences because it did not force a particular strategy on the children. We per- mitted the children to search until they found the sticker, which meant that children who adopted strategies such as serial searching or beaconing still succeeded in finding the sticker on each trial, albeit less efficiently than by using a middle rule. We are currently investigating whether limiting the number of searches per trial influences the emergence of sex differences. Regardless of the reason, however, our results are important because they clearly show a sex difference in the spatial search behavior of preschool children.

\section{REFERENCES}

Cartwright, B. A., \& Collett, T. S. (1983). Landmark learning in bees. Journal of Comparative Physiology, 151A, 521-543.

Cheng, K., \& Spetch, M. L. (1998). Mechanisms of landmark use in mammals and birds. In S. Healy (Ed.), Spatial representation in animals (pp. 1-17). Oxford: Oxford University Press.

Choi, J., \& Silverman, I. (2003). Processes underlying sex differences in route-learning strategies in children and adolescents. Personality \& Individual Differences, 34, 1153-1166.

Collett, T. S., Cartwright, B. A., \& Smith, B. A. (1986). Landmark learning and visuo-spatial memories in gerbils. Journal of Comparative Physiology, 158A, 835-851.

Duffy, S., HutTenlocher, J., \& Levine, S. (2005). It is all relative: How young children encode extent. Journal of Cognition \& Development, 6, 51-63.

GEARY, D. C. (1996). Sexual selection and sex differences in mathematical abilities. Behavioral \& Brain Sciences, 19, 229-284.

Goldin-Meadow, S. (1997). When gestures and words speak differently. Current Directions in Psychological Science, 6, 138-143.

Jones, C. M., Braithwaite, V. A., \& Healy, S. D. (2003). The evolution of sex differences in spatial ability. Behavioral Neuroscience, 117, 403-411.

Jones, J. E., Antoniadis, E., Shettleworth, S. J., \& Kamil, A. C. (2002). A comparative study of geometric rule learning by nutcrackers (nucifraga columbiana), pigeons (columba livia), and jackdaws (corvus manedula). Journal of Comparative Psychology, 116, 350-356.

Kamil, A. C., \& Jones, J. E. (1997). The seed-storing corvid Clark's nutcracker learns geometric relationships among landmarks. Nature, 390, 276-279.

Levine, S. C., Huttenlocher, J., Taylor, A., \& Langrock, A. (1999). Early sex differences in spatial skill. Developmental Psychology, 35, 940-949.

MacDonald, S. E., Spetch, M. L., Kelly, D. M., \& Cheng, K. (2004). Strategies in landmark use by children, adults, and marmoset monkeys. Learning \& Motivation, 35, 322-347.

McGuinness, D., \& Morley, C. (1991). Sex differences in the development of visuo-spatial ability in pre-school children. Journal of Mental Imagery, 15, 143-150.

Poti, P., Bartolommei, P., \& Saporiti, M. (2005). Landmark use by Cebus apella. International Journal of Primatology, 26, 921-948.

Rosser, R. A., Ensing, S. S., Gilder, P. J., \& Lane, S. (1984). An information-processing analysis of children's accuracy in predicting the appearance of rotated stimuli. Child Development, 55, 22042211 .

Solomon, T. L., \& Levine, S. C. (2005, June). Young children's ability to use landmark configurations. Paper presented at the Society for Research in Child Development Meeting, Atlanta.

Spetch, M. L., Cheng, K., \& MacDonald, S. E. (1996). Learning the configuration of a landmark array: I. Touch-screen studies with pigeons and humans. Journal of Comparative Psychology, 110, 55-68.

Spetch, M. L., Cheng, K., MacDonald, S. E., LinkenhoKer, B. A., Kelly, D. M., \& Doerkson, S. R. (1997). Use of landmark configuration in pigeons and humans: II. Generality across search tasks. Journal of Comparative Psychology, 111, 14-24.

Spetch, M. L., \& Kelly, D. M. (2006). Comparative spatial cognition: Processes in landmark- and surface-based place finding. In E. A. Was- 
serman and T. R. Zentall (Eds.), Comparative cognition: Experimental explorations of animal intelligence. New York: Oxford University Press.

Sutton, J. E., Olthof, A., \& Roberts, W. A. (2000). Landmark use by squirrel monkeys (Saimiri sciureus). Animal Learning \& Behavior, 28, 28-42.

Uttal, D. H., Gregg, V. H., Tan. L. S., Chamberlin, M. H., \& Sines, A (2001). Connecting the dots: Children's use of a systematic figure to facilitate mapping and search. Developmental Psychology, 37, 338350 .

Uttal, D. [H.], \& Newcombe, N. S. (2005, June). One hidden land- mark, two spatial codes: The development of distance and relational coding. Paper presented at the Society for Research in Child Development Meeting, Atlanta.

Vasilyeva, M., \& Huttenlocher, J. (2004). Early development of scaling ability. Developmental Psychology, 40, 682-690.

Voyer, D., Voyer, S., \& Bryden, M. P. (1995). Magnitude of sex differences in spatial abilities: A meta-analysis and consideration of critical variables. Psychological Bulletin, 117, 250-270.

(Manuscript received July 20, 2005;

revision accepted for publication February 21, 2006.) 